\title{
INSEGURANÇA ALIMENTAR E NUTRICIONAL DE FAMÍLIAS COM CRIANÇAS MENORES DE CINCO ANOS: FATORES ASSOCIADOS
}

\author{
Claudia Moreira de Lima ${ }^{1}$ \\ Rayane Kelly Fernandes da Costa ${ }^{2}$ \\ Patrycia Conceição de Almeida Costa ${ }^{3}$ \\ Dayane dos Santos Souza Magalhães ${ }^{4}$ \\ Dennislaine Alves Lima Dantas ${ }^{5}$ \\ Grasiela Cristina Silva Botelho Silvestre ${ }^{6}$
}

RESUMO: Segurança alimentar e nutricional, significa dar garantia e condições de acesso a alimentos básicos de qualidade a todos, em quantidade suficiente. Objetivo: Assim, o presente estudo tem como objetivo avaliar a segurança alimentar de famílias com crianças de o a 5 anos por meio do método da Escala Brasileira de Insegurança (EBIA), sendo o indicador sensível para detectar famílias em risco de insegurança alimentar (IA). Metodologia: Trata-se de um estudo com um desenho transversal, com abordagem quantitativa com delineamento descritivo/exploratório. Resultados: Das 68 crianças avaliadas, $56(82,35 \%)$ pertenciam à faixa etária de o a 3 anos incompletos e 12 (17,65\%) compreendiam a idade entre 3 a 5 anos, com média de idade de or \pm 04 anos. Observou-se, na distribuição por sexo, uma igualdade entre os participantes tendo para o sexo feminino 34 (50\%) e o masculino 34 (50\%), observa-se que foi predominante (93,1\%) os domicílios com renda familiar mensal menor que dois salários mínimos e $58,1 \%$ das famílias eram constituídas por até quatro moradores. Conclusão. Os resultados deste estudo indicam que existe pouca família em estado de insegurança alimentar, estando associada, principalmente, ao baixo nível socioeconômico das famílias.

Descritores: Escala Brasileira de Insegurança. Segurança Alimentar. Família.

\footnotetext{
I Enfermeira Mestra, pelo Programa de Pós-Graduação em Ambiente e Saúde da Universidade de Cuiabá (UNIC). Professora Assistente da Universidade do Estado de Mato Grosso - UNEMAT, Diamantino, Mato Grosso-Brasil.

${ }_{2}$ Graduanda em Enfermagem pela Universidade do Estado de Mato Grosso - UNEMAT, Diamantino, Mato Grosso-Brasil

${ }^{3}$ Graduanda em Enfermagem pela Universidade do Estado de Mato Grosso - UNEMAT, Diamantino, Mato Grosso-Brasil.

${ }^{4}$ Enfermeira Mestra, pelo Programa de Pós-Graduação em Ambiente e Saúde da Universidade de Cuiabá (UNIC). Enfermeira assistencial na Empresa Brasileira deServiços Hospitalares no Hospital Universitário Júlio Muller da Universidade Federal doMato Grosso/UFMT/EBSERH.

5 Enfermeira assistencial da Clínica Pediátrica no Hospital Regional Dro Antônio Fontes. Pós-Graduada Latu Sensu em Enfermagem do Trabalho e Saúde do Trabalhador e Gestãoem Saúde.

${ }^{6}$ Enfermeira Mestra, pelo Programa de Pós-Graduação em Ambiente e Saúde da Universidade de Cuiabá (UNIC). Docente Assistente da Universidade do Estado de MatoGrosso (UNEMAT), Campus Universitário de Diamantino/MT
} 
ABSTRACT: Food and nutrition security means ensuring and providing sufficient access to quality staple food to all. Objective: thus, this study aims to evaluate the food security of families with children from o to 5 years old using the Brazilian Insecurity Scale (EBIA) method, being the sensitive indicator to detect families at risk of food insecurity (AI). Methodology: This is a cross-sectional study with a quantitative approach with a descriptive/ exploratory design. Results: of the 68 children evaluated, 56(82.35\%) belonged to the age group from o to 3 years old incomplete and 12 (17.65\%) comprised the age between 3 to 5 years, with na average age of or the 04 years. It was observed, in the distribution by sex, an equality between the participants having for the female sex 34 (50\%) and the male $34(50 \%)$, it is observed that the households with Family income were predominant (93.1\%) less than two minimum wages and $58.1 \%$ of families consisted of up to four residents. Conclusion: the results of this study indicate that there is little family in a state of food insecurity, being mainly associated with the low socioeconomic level of the families.

Keywords: Brazilian Insecurity Scale. Food Security. family.

\section{INTRODUÇÃO}

A fome encontra-se na lista dos dez maiores riscos à saúde, sendo esta situação considerada o maior problema solucionável do mundo (FACCHINI et. al., 2014). Embora a segurança alimentar e nutricional (SAN), constitua-se como um direito fundamental, estudo das Organizações das Nações Unidas (ONU), estimou que uma a cada ro pessoas (9,3\% ou 689 milhões de pessoas), de aproximadamente 150 países, se encontrava em insegurança alimentar e nutricional (IAN), grave ou fome, segundo dados de 2014, 2015 e 2016 (ONU, 2017).

Segundo o panorama da ONU as desigualdades sociais e econômicas se refletem no déficit de crescimento de crianças. Em Honduras, o problema afeta $42 \%$ das crianças em famílias de baixa renda e apenas $8 \%$ das crianças de famílias de renda mais alta. $\mathrm{Na}$ Guatemala, a diferença é maior: o déficit de crescimento afeta $66 \%$ das crianças mais pobres e I7\% das crianças de família de renda mais alta (ONU, 2018). No que concerne o Brasil, tem-se que provavelmente $17,5 \%$ de famílias com crianças menores de cinco anos se encontram em situação de IAN moderada/grave, com prevalências concentradas em famílias de classe econômica baixa (34\%) e em beneficiários de programas de transferência de renda $(36,5 \%)$ (POBLACION et. al., 2014; SANTOS et al, 2018a).

A desnutrição em crianças menores de cinco anos afeta de maneira direta o desenvolvimento cognitivo, aumentar o risco de infecção, o desenvolvimento de doenças crônicas, e até o crescimento econômico do país, além de ser considerada fator subjacente de 
mortes em menores de um ano de idade. Estando a IAN ligada às condições socioeconômicas ambientais, maternas, relacionadas às práticas alimentares infantis, morbidades e de acesso aos serviços de saúde (PEDRAZA; GAMA, 2015; ARAÚJO et al, 2016).

Assim a IAN pode ser vista tanto em situações de alimentação insuficiente, que podemos caracterizar como fome e desnutrição, quanto em situações de doenças decorrentes de uma alimentação inadequada, como o excesso de peso e carências específicas de nutrientes corporais (TRIVELLATO et al., 2019).

Entre os indicadores comumente utilizados no meio científico para avaliação da SAN, destacam-se as escalas de percepção da (in) segurança alimentar, os de produção e consumo de alimentos, os antropométricos, socioeconômicos, bioquímicos e clínicos (SPERANDIO et al, 20I8a). Assim, temos a Escala Brasileira de Insegurança Alimentar (EBIA), como indicador sensível para detectar famílias em risco de (in) segurança alimentar além de possibilitar uma avaliação dos efeitos das políticas públicas, sendo este um instrumento validado e adaptado para a população brasileira, contemplando as especificidades e as diversidades nacionais, podendo ser aplicado tanto nas zonas rurais como na urbana (PÉREZ-ESCAMILLA et. al., 2004b; SPERANDIO et al, 2015b; RADIMER et. al., 2015; BEZERRA et al., 2017).

Portanto, diante dos dados crescentes de problemas relacionados a questão nutricional no Brasil, o presente estudo teve como objetivo investigar a prevalência de insegurança alimentar e fatores associados de famílias com crianças de o a 5 anos de idade atendidas por Estratégias de Saúde da Família de um município do interior de Mato Grosso, Brasil.

\section{METODOLOGIA}

Estudo de delineamento transversal desenvolvido no contexto das unidades de Estratégias de Saúde da Família -ESF’s de município da região médio norte do estado de Mato Grosso, Brasil. Constitui a população deste estudo crianças até cinco anos de idade, assim como suas famílias.

Como variáveis independentes utilizadas na análise deste estudo, investigou-se as variáveis demográficas (sexo, idade e número de indivíduos no domicílio), variáveis socioeconômicas (renda familiar mensal, escolaridade do chefe da família e escolaridade dos adolescentes), variáveis relativas às condições de moradia e saneamento (tipo de construção 
da moradia, coleta de lixo, abastecimento e tratamento de água e esgoto sanitário), medidas antropométricas (peso e estatura das crianças para cálculo do IMC) e de segurança alimentar e nutricional (participação em programas sociais do governo de transferência direta de renda).

A avaliação do perfil antropométrico foi realizada por meio da aferição de peso (kg) e estatura $(\mathrm{cm})$, após aferidos o peso e estatura, esses dados foram registrados em uma planilha no Excel. A aferição do peso foi feita com auxílio de uma balança eletrônica (digital) G-Tech ${ }^{\circledR}$ modelo Glass 8 em crianças menores de 2 anos de idade.

Em crianças de 2 a 5 anos de idade o peso foi aferido em balança plataforma portátil, eletrônica, marca Bioland EF934, capacidade para 20okg e sensibilidade de roog, onde neste caso a criança foi colocada no centro do equipamento, usando uniforme da escola, descalça, ereta, com os pés juntos e os braços estendidos ao longo do corpo. Após mantê-la parada nessa posição e o valor do peso estar fixado no visor, realizou-se a leitura do mesmo (BRASIL, 2008b).

Para a aferição da estatura adota-se o termo "comprimento" para a estatura de crianças menores de 2 anos, que devem ser medidas deitadas, e o termo "altura" para a estatura de crianças maiores de 2 anos, adolescentes, adultos, idosos e gestantes, que devem ser medidos em pé (BRASIL, 20IIc). Para as crianças menores de dois anos para mensurar o comprimento foi utilizado estadiômetro portátil, com trena retrátil, de extensão até $200 \mathrm{~cm}$ com precisão de o, I cm.

Crianças com idade menor que 24 meses foram aferidas, com auxílio do antropometro infantil com capacidade de até roscentímetros $(\mathrm{cm})$, com escala milimétrica, estando as crianças deitadas sobre uma mesa. Para crianças acima de dois anos a altura foi aferida utilizando-se fita métrica com capacidade para I,5m e sensibilidade de $1,0 \mathrm{~mm}$, fixada em parede sem rodapé a uma altura de $150 \mathrm{~cm}$ do piso.

Essa medida foi realizada com a criança em pé, descalça, com a cabeça livre de adereços, posição ereta, braços estendidos ao longo do corpo, cabeça erguida, olhando para um ponto fixo na altura dos olhos, pernas e pés unidos, além de calcanhares, panturrilhas, glúteos, escápulas e parte posterior da cabeça (região do occipital), encostados na parede (BRASIL, 2008b). Quando não foi possível encostar os cinco pontos, procurou-se encostar pelo menos três deles. 
Nesta pesquisa, para estabelecer o estado nutricional das crianças o índice utilizado foi o Peso para Altura (P/A) e os valores dos escores $z$ foram obtidos com o auxílio do software WHO Anthro, que calculou os escores-z utilizando o peso e a altura de cada criança (BRASIL, 2oIIc; DAWALIBI et al., 20II).

Para a determinação da classificação nutricional da amostra estudada, após os cálculos, o estado nutricional das crianças foi classificado utilizando-se o escore-z, tendo como parâmetro os pontos de corte de P/A estabelecidos pelas Novas Curvas da OMS/2006, recomendados pelo Ministério da Saúde -MS (BRASIL, 20Irb; BRASIL, 2009c).

Utilizando o critério estatístico de escore-z e a classificação recomendada pelo MS, crianças menores de cinco anos foram classificadas com desnutrição quando o IMC/I for $z$ $<-2$; eutrofia quando o escore para IMC/I for $z>-2$ a $z<+2$; excesso de peso quando o escore para IMC/I for $z>+2$ (BRASIL, 20Ib; BRASIL, 2009c).

Considerou-se como variável dependente a situação de insegurança alimentar, e para mensurá-la aplicou-se a EBIA para identificação e classificação das famílias. A EBIA é composta por I4 perguntas centrais dicotômicas - Sim ou Não, nos últimos três meses (IBGE, 2006a; SEGALL-CORREA, 2009), sendo que cada resposta afirmativa representa um ponto e a somatória desses pontos avalia a insuficiência alimentar em diferentes níveis de intensidade, com pontuações diferentes para domicílios com pelo menos um morador de menos de 18 anos de idade e domicílios com somente moradores de 18 anos ou mais de idade (IBGE, 2006a).

A EBIA está dividida em quatro níveis, a saber: No nível de segurança, todas as perguntas são respondidas negativamente, temos zero de respostas positivas. $\mathrm{Na}$ insegurança leve, de uma a cinco perguntas são respondidas afirmativamente, e nesse nível o aspecto mais afetado é a qualidade da alimentação, juntamente com a preocupação de que possa faltar alimento no futuro próximo. $\mathrm{Na}$ insegurança moderada, temos de seis a dez respostas positivas; começa a haver restrição quantitativa na alimentação dos adultos da família. Por último, na insegurança grave, temos de onze a quinze respostas afirmativas ao questionário - aqui aparece deficiência quantitativa e mesmo fome entre adultos e crianças da família (IBGE, 2006a).

Visto que, a situação de segurança alimentar se apresenta dentro uma multicausalidade com diversos fatores de risco que podem ser classificados em duas 
dimensões: Dimensão Domiciliar e Dimensão Individual, tendo estes fatores distais e proximais. Na Dimensão Domiciliar, podemos encontrar informações do domicílio e características familiares e como fatores distais temos as características das condições de moradia e saneamento, participação em programas sociais do governo de transferência direta de renda (Bolsa Família); e como fatores que atuam diretamente no evento tem-se os fatores proximais: as características socioeconômicas e a disponibilidade de alimentos (GUERRA, 20II).

$\mathrm{Na}$ Dimensão Individual, encontram-se às informações individuais das crianças sobre o estado nutricional e o consumo alimentar. Nesta dimensão estão os fatores que possivelmente podem sofrer influência da situação de insegurança alimentar e nutricional, bem como, dos fatores da Dimensão Domiciliar.

As informações contidas nos questionários e no banco de dados utilizadas neste estudo foram duplamente digitados a fim de verificar possíveis falhas e inconsistências e visando a confiabilidade psicométrica da Escala Brasileira de Insegurança aplicada nesse estudo, sendo calculado o Alpha de Crombach3.

Para a análise do banco de dados, gerado da tabulação dos dados, foi utilizado o programa Prisma Graph 5.0, sendo os resultados analisados de forma quantitativa, e apresentados por meio de gráfico e tabelas.

Os resultados foram descritos em valores médios, variância, desvios-padrão e frequências absolutas e relativas (\%). Após análise descritiva das variáveis, avaliou-se a relação das variáveis explicativas com a variável resposta (sem sobrepeso ou obesidade e com sobrepeso ou obesidade) por meio do teste de associação de Pearson. O nível de significância estatística considerado foi de $5 \%(\mathrm{p}<0,05)$.

O projeto de pesquisa foi aprovado pelo Comitê de Ética em Pesquisa da Universidade de Cuiabá (UNIC). Foram respeitados os preceitos éticos e legais de pesquisas que envolvem seres humanos de acordo com a resolução 466/12, assim como a resolução 3II/2007 do Conselho Federal de Enfermagem.

\section{RESULTADOS}

Foram avaliadas 68 crianças com idade até 5 anos agrupadas em faixas etárias, de o a menores de 3 anos e de 3 a 5 anos, de ambos os sexos, atendidas pelas ESF's em que o estudo foi realizado. 
Das 68 crianças avaliadas, $56(82,35 \%)$ pertenciam à faixa etária de o a 3 anos incompletos e 12 (17,65\%) compreendiam a idade entre 3 a 5 anos, com média de idade de or \pm o4anos. Observou-se, na distribuição por sexo, uma igualdade entre os participantes tendo para o sexo feminino 34 (50\%) e o masculino 34 (50\%) (Tabela I).

Tabela I - Distribuição das crianças, segundo variáveis sexo e idade, Diamantino, Mato Grosso, 2020.

\begin{tabular}{cccc}
\hline \hline Variáveis & Categorias & $\mathrm{N}$ & $\%$ \\
\hline \hline \multirow{2}{*}{ Sexo } & $(\mathrm{n}=68)$ & & \\
& masculino & 34 & 50,00 \\
& feminino & 34 & 50,00 \\
Idade & $0-<3$ & 56 & 82,35 \\
& $3-5$ & 12 & 17,65
\end{tabular}

Fonte: dados da pesquisa. Elaboração: próprio autor.

Observa-se (Tabela 2) que foi predominante (93,1\%) os domicílios com renda familiar mensal menor que dois salários mínimos e 58,1\% das famílias eram constituídas por até quatro moradores.

Tabela 2 - Distribuição da renda, número de moradores, informações de moradia/saneamento e participação em programas sociais, dos participantes da pesquisa, Diamantino, Mato Grosso, 2020.

\begin{tabular}{|c|c|c|c|}
\hline Variáveis & Categorias & $\mathbf{N}$ & $\%$ \\
\hline & $(n=68)$ & & \\
\hline Renda familiar mensal & Até i Salário mínimo & $2 \mathrm{I}$ & 30,88 \\
\hline (salário mínimo)* & Mais de I Salários mínimo & 47 & 69,12 \\
\hline \multirow[t]{3}{*}{ № de moradores no domicílio } & até 4 & 20 & $29,4 \mathrm{I}$ \\
\hline & $5-6$ & 34 & 50,00 \\
\hline & 7 ou mais & 14 & 20,59 \\
\hline \multirow[t]{2}{*}{ Tipo de moradia } & Casa própria & 52 & 76,47 \\
\hline & Casa alugada ou cedida & I6 & 23,53 \\
\hline Abastecimento de água & rede pública & 68 & 100,00 \\
\hline
\end{tabular}




\begin{tabular}{llll}
\hline Participação em programas sociais do & sim & 04 & 5,88 \\
governo & não & 04 & 5,88 \\
\hline TOTAL & & 64 & $100 \%$ \\
\hline
\end{tabular}

Fonte: Dados da pesquisa. Elaboração: próprio autor.

Em relação às características de moradia e saneamento, a maior parte dos entrevistados $(76,47 \%)$ respondeu ter moradia própria. Das 68 famílias ıoo\% eram atendidos pela coleta pública de lixo e apresentavam rede pública de esgoto sanitário em suas residências. $\mathrm{O}$ abastecimento de água era distribuído por meio da rede pública a todos as famílias participantes (เоo\%).

\section{SITUAÇÃO DA SEGURANÇA ALIMENTAR E NUTRICIONAL}

A Escala Brasileira de Insegurança Alimentar (EBIA) evidenciou uma consistência interna muito boa, visto que o Alpha de Crombach foi de o,92.

Conforme apresentado na Figura I, observou-se que a situação de segurança alimentar foi identificada em 72,06\% das famílias estudadas. Desta Forma, 27.94\% das famílias estavam em situação de insegurança alimentar e nutricional, e segundo os seus níveis de severidade: $16,18 \%$ estavam em insegurança leve, 8,82 \% insegurança moderada e 2,94\% em insegurança grave.

Figura I - Representação da situação de segurança alimentar e nutricional das famílias de crianças menores de cinco anos atendidas pela atenção primaria. Diamantino - MT, Brasil. 2020.

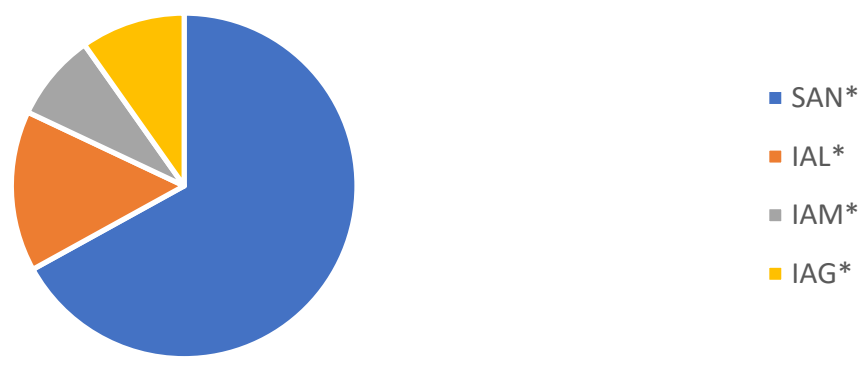

Fonte: Dados da pesquisa. Elaboração: próprio autor. 
Níveis de divisão da EBIA conforme resultados. SAN: segurança alimentar e nutricional; IAL: insegurança alimentar leve; IAM: insegurança alimentar moderada; IAG: insegurança alimentar grave.

Quando observada a frequência de respostas positivas para as perguntas da EBIA, obteve-se maior frequência para as perguntas I "teve preocupação que a comida acabasse antes que pudesse comprar ou receber mais", 3 "ficou sem dinheiro para ter uma alimentação saudável e variada", 4 "ficou sem dinheiro e teve que se arranjar com apenas alguns alimentos disponíveis" e 2 "ficou sem dinheiro e sem alimentos disponíveis" e menores frequências para as perguntas 8 "maiores de 18 anos tiveram fome, por escassez de comida no dia" e Io "menores de 18 anos ficaram sem comer o suficiente por falta de dinheiro". Os dados referentes as repostas positivas podem ser mais bem visualizados na figura 02 .

Figura 02 - Frequência de respostas positivas em ordem crescente para as 14 perguntas da EBIA das famílias de crianças menores de cinco anos atendidas pela atenção primaria. Diamantino - MT, Brasil. 2020.

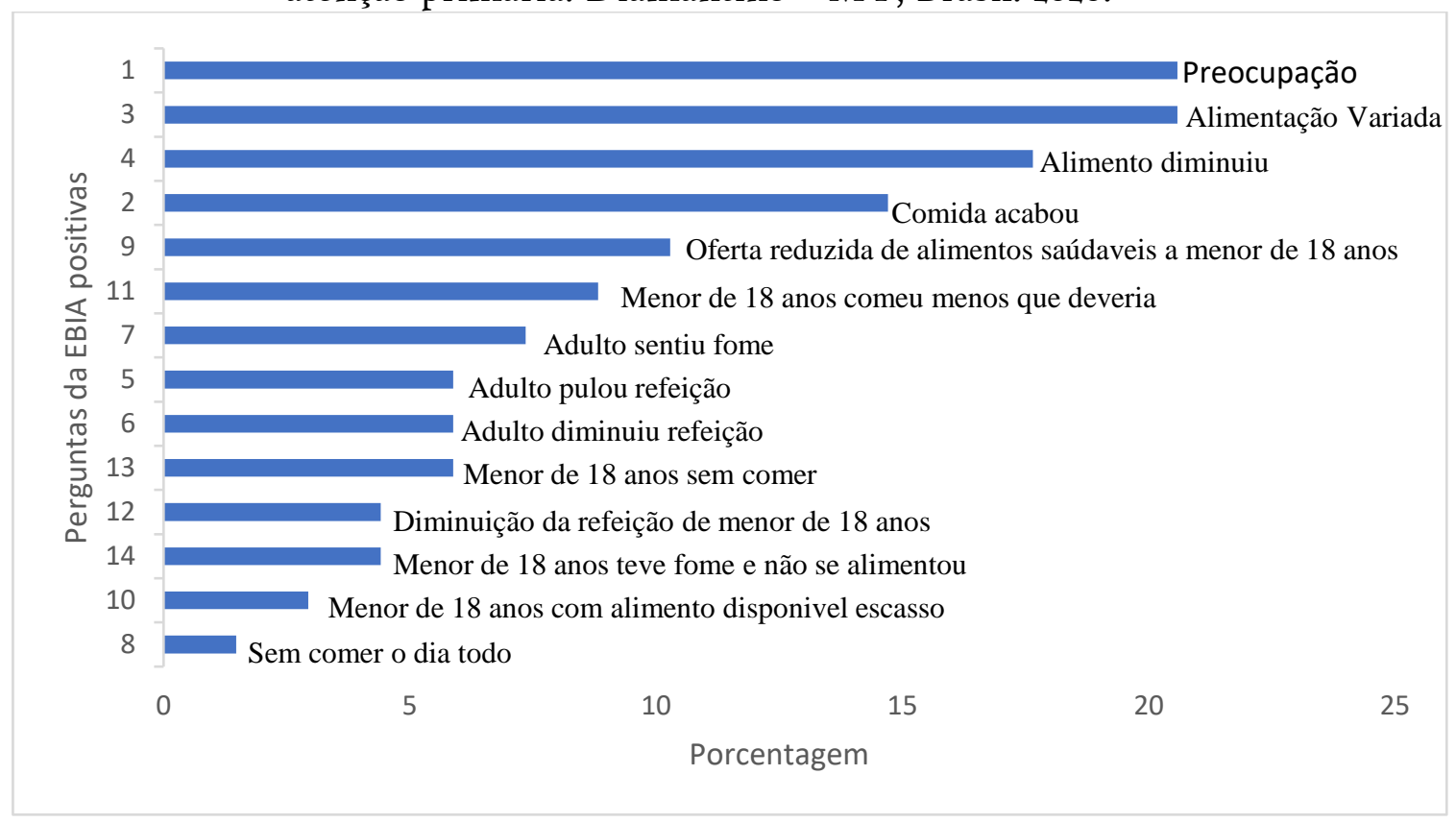

Fonte: Dados da pesquisa. Elaboração: próprio autor.

\subsection{FATORES ASSOCIADOS À SITUAÇÃO DE INSEGURANÇA ALIMENTAR E NUTRICIONAL}

A análise estatística dos dados foi realizada por meio de blocos. As famílias foram classificadas em dois grupos de acordo com a situação de segurança alimentar identificada na EBIA. As famílias que apresentaram alguma resposta positiva ao questionário, foram 
agrupadas e geraram a variável IAN, e as famílias que não tiveram nenhuma resposta positiva a uma das quatorze perguntas da EBIA foram agrupadas gerando a variável SAN, sendo 72,06\% pertencentes ao grupo SAN e 27,94\%, com IAN.

Os resultados a seguir, tratam da relação entre a situação de segurança alimentar das famílias e variáveis de sexo e idade, e variável relativa ao estado nutricional das crianças (Tabela 3).

Tabela 3 - Prevalência, razão de prevalência, intervalo de confiança e p-valor da situação de segurança alimentar e nutricional segundo informações das crianças menores de cinco anos das famílias pesquisadas, atendidas pela atenção primaria. Diamantino - MT, Brasil. 2020.

\begin{tabular}{|c|c|c|c|c|c|c|c|c|}
\hline \multirow[t]{2}{*}{ Variáveis } & \multirow[t]{2}{*}{ Categorias } & \multicolumn{2}{|c|}{ IAN* } & \multicolumn{2}{|c|}{ SAN* } & \multirow[t]{2}{*}{ RP* } & IC* & \multirow{2}{*}{$\begin{array}{l}\text { p- } \\
\text { valor* } \\
\left(\chi^{2}\right)\end{array}$} \\
\hline & & $\mathbf{N}$ & $\%$ & $\mathbf{N}$ & $\%$ & & $(95 \%)$ & \\
\hline \multicolumn{9}{|c|}{ Informações da } \\
\hline criança & $(n=68)$ & & & & & & & \\
\hline \multirow[t]{2}{*}{ Sexo } & masculino & 13 & 68,42 & $2 \mathrm{I}$ & 42,86 & 0,99 & - & 0,049 \\
\hline & feminino & o6 & 31,58 & 28 & 57,14 & 0,99 & $\mathrm{o}, 40-0, \mathrm{I}$ & \\
\hline \multirow[t]{3}{*}{ Idade } & $0-<3$ & 12 & 63,16 & 43 & 87,76 & 0,99 & - & \\
\hline & $3-5$ & 07 & 36,84 & 6 & 12,24 & 0,98 & $0,09-0,3$ & \\
\hline & & & & & & & & 0,021 \\
\hline Estado & $(n=68)$ & & & & & & & \\
\hline \multirow[t]{5}{*}{ Nutricional } & Eutrófico & Iо & 52,63 & IO & $20,4 \mathrm{I}$ & 0,99 & - & \\
\hline & Baixo peso & 03 & 15,79 & 15 & 30,6r & 0,99 & 0,05 & 0,093 \\
\hline & & & & & & & 0,30 & \\
\hline & Sobrepeso/obeso & o6 & 31,58 & 24 & 48,98 & 0,99 & 0,09 & \\
\hline & & & & & & & 0,12 & \\
\hline
\end{tabular}

*IAN: insegurança alimentar e nutricional; SAN: segurança alimentar e nutricional; RP: razão de prevalência; IC: intervalo de confiança; $p$-valor: Qui-quadrado de Pearson.

Conforme apresentado na Tabela 03, verificou-se relação entre sexo da criança e a idade com a situação de insegurança alimentar. A prevalência de IAN nas crianças do sexo 
masculino foi de 68,42\% (p=o,049) e de 31,58\% nas meninas. Quanto a idade as crianças com IAN, apresentaram prevalência de insegurança alimentar de 63,16\% (p=o,o2I) e de 36,84\%, respectivamente.

Por meio da Tabela 4, foi identificado relação significante estatisticamente entre a insegurança alimentar e número de moradores, entretanto nenhuma outra relação estatisticamente significativa foi encontrada considerando as demais variáveis (tipo de moradia, participação em programas sociais do governo e renda familiar mensal).

Tabela 4 - Prevalência, razão de prevalência, intervalo de confiança e p-valor da situação de segurança alimentar e nutricional segundo variáveis sociodemográficas e variáveis relacionadas as famílias de crianças menores de cinco anos pesquisadas, atendidas pela atenção primaria. Diamantino - MT, Brasil. 2020.

\begin{tabular}{|c|c|c|c|c|c|c|c|c|}
\hline \multirow[t]{2}{*}{ Variáveis } & \multirow[t]{2}{*}{ Categorias } & \multicolumn{2}{|c|}{ IAN* } & \multicolumn{2}{|c|}{ SAN* } & \multirow[t]{2}{*}{ RP* } & \multirow[t]{2}{*}{ IC* } & \multirow{2}{*}{$\begin{array}{l}p \text {-valor } \\
2^{*}\end{array}$} \\
\hline & & $\mathrm{N}$ & $\%$ & $\mathrm{~N}$ & $\%$ & & & \\
\hline № de moradores no & até 4 & 07 & 36,84 & 13 & 26,53 & $\mathrm{I}, \mathrm{OO}$ & $2,00-2,35$ & 0,280 \\
\hline \multirow[t]{2}{*}{ domicílio } & $5-6$ & 12 & 63,16 & 22 & 44,90 & 0,99 & $\mathrm{I}, \mathrm{OO}-2,4 \mathrm{I}$ & 0,032 \\
\hline & 7 ou mais & oo & o & 14 & 28,57 & 0,98 & $2,29-2,87$ & 0,511 \\
\hline \multirow[t]{2}{*}{ Tipo de moradia } & Casa própria & 15 & 78,95 & 38 & 77,55 & 0,99 & $2,47-3,65$ & o,90I \\
\hline & Casa alugada ou cedida & 04 & 21,05 & II & 22,45 & 0,99 & $2,05-2,22$ & 0,896 \\
\hline Participação em & não & I8 & 94,74 & 46 & 93,88 & I & $2,65-3,98$ & \\
\hline programas sociais $\mathrm{dc}$ & osim & oI & 5,26 & 03 & 6,12 & 0,89 & $6,35-2,48$ & 0,908 \\
\hline \multicolumn{9}{|l|}{ governo (bolsa família) } \\
\hline Renda familiar mensal & Até I salário mínimo & 13 & 26,53 & 09 & 47,37 & 0,99 & - & o,IIS \\
\hline \multirow[t]{2}{*}{ (Salário mínimo@) } & Até 3 salários mínimo & 26 & 53,06 & 07 & 36,84 & 0,99 & $3,08-1,99$ & 0,319 \\
\hline & $\begin{array}{l}4 \text { ou mais salários } \\
\text { mínimos }\end{array}$ & Io & $20,4 \mathrm{I}$ & 03 & 15,79 & 0,98 & $2,16-2,48$ & 0,382 \\
\hline
\end{tabular}

*IAN: insegurança alimentar e nutricional; SAN: segurança alimentar e nutricional; RP: razão de prevalência; IC: intervalo de confiança; *p-valor 2: Qui-quadrado de Pearson@

A prevalência de IAN foi de $26,53 \%$ em famílias com renda menor ou igual a i salário mínimo, sendo que, ter uma renda familiar mensal menor ou igual a s salário mínimo neste 
estudo não foi caracterizado como um possível fator de risco para a insegurança alimentar no domicílio.

Em famílias com a moradia própria, a prevalência de IAN foi de 78,95\% (IC95\%= $2,47-3,65)$. Portanto, domicílios com tais características apresentaram-se neste estudo como improvável fator de risco para situação de insegurança alimentar.

Para os domicílios que informaram participar de programas sociais do governo, a prevalência de IAN foi igual a 5,26\% (IC95\% = 6,35-2,48).

As estimativas das razões de prevalência (RP), comparadas as variáveis que se mostraram associadas à insegurança alimentar (Tabela 3 e 4), mostrou que sexo, idade e número de moradores onde reside foram os indicadores que melhor discriminaram as famílias em risco de insegurança alimentar. Pois, todas essas variáveis apresentaram p-valor menor 0,05 .

\section{DISCUSSÃO}

Os resultados apresentaram uma consistência interna elevada, visto que o Alpha de Cronbach foi de 0,92 , demonstrando que as perguntas foram respondidas de acordo com a expectativa teórica própria da escala, em ordem crescente de gravidade da insegurança alimentar.

Nesta perspectiva, o presente estudo realizou a análise descritiva, tendo assim o número mediano de respostas positivas às questões da EBIA de 6,36 questões. Um total de 49 respondentes não pontuaram em qualquer um dos questionamentos da escala e essa quantidade expressa que $72,06 \%$ das famílias não apresentavam no momento desta pesquisa qualquer grau de IAN. Enquanto a prevalência de IAN foi de que $27.94 \%$ das famílias estavam em situação de insegurança alimentar e nutricional, e segundo os seus níveis de severidade: $16,18 \%$ estavam em insegurança leve, $8,82 \%$ insegurança moderada e 2,94\% em insegurança grave.

Nossos resultados se aproximam da última pesquisa realizada em 2013 no Brasil, onde a prevalência de IA foi estimada em 22,6\% dos domicílios com IA, sendo destes $14,8 \%$ para grau leve, 4,6\% grau moderado e 3,2\% em grave situação de IA (IBGE, 2013b).

Analisando a EBIA com relação a frequência observada de respostas positivas às quatorze perguntas do questionário, a primeira e a terceira questões apresentaram maior percentual de concordância alcançando $31,46 \%$ de respostas positivas. $O$ que infere 
preocupação quanto a comida disponível em domicilio acabar e não ter financeiro para adquirir o mesmo, o que vai de encontro com estudo realizado na Bahia, onde o indicador ambiental se mostrou com associação positiva e significante (RIBEIRO et al., 2018).

Nesta perspectiva, o Brasil representa uma situação conflitante, na medida em que, sendo um dos maiores produtores de alimentos do mundo, ainda convive com uma condição social em que milhões de pessoas se encontram à margem da cidadania, por não terem assegurado, entre outros, o direito básico à alimentação suficiente, completa, adequada e saudável (OLIVEIRA et al, 2009).

Estando a situação de restrição quantitativa e qualitativa de alimentos associada à renda insuficiente, onde quanto maior a proporção de domicílios em condições de insegurança alimentar menor é o rendimento familiar (COTTA e MACHADO, 2013b), uma vez que o acesso a aos alimentos também é influenciado pelo preço, determinando quantidade e qualidade dos alimentos, uma vez que produtos caloricamente densos costumam ser mais baratos que os alimentos tradicionais comparados aos custos de uma alimentação saudável (ANTUNES, 2018).

Corroboram com estes dados estudos que apontam como causas subjacentes das diversas formas de inadequações nutricionais as rápidas mudanças ocorridas no padrão alimentar a partir da década de 8o, com um aumento significativo pela preferência por alimentos ultra processados em âmbito mundial (MARTINS et al, 2013; FAO, OPAS, 2017), dados que foram identificados em estudo realizado no Brasil, onde evidenciou o declínio no consumo de alimentos básicos, com uma evolução da disponibilidade domiciliar de alimentos no Brasil (IBGE, 20I0c).

Diante disso, uma potente função da política de alimentação é apoiar um ambiente que incentive o aprendizado de preferências alimentares, além de atuar na superação de barreiras como custo dos alimentos mais saudáveis, indisponibilidade qualitativa e quantitativa de alimentos, mudanças nos papéis sociais e organizacionais das famílias gerando falta de tempo para o preparo de refeições em casa (HAWKES, 2015).

Assim, neste estudo analisou-se a relação da situação de insegurança alimentar com informações das crianças como sexo, idade e condição nutricional e condições socioeconômicas das suas famílias, avaliando as informações relativas aos domicílios - tipo de moradia; renda familiar mensal e a participação em programas sociais do governo. 
Das crianças participantes deste estudo, observou-se prevalência de IAN maior nos meninos $(68,42 \%)$ que nas meninas $(31,58 \%)$. Com relação a este achado, os principais determinantes de IAN mundialmente reconhecidos são os fatores econômicos (renda familiar, programas governamentais), domiciliares (moradia, família) e sociais adversos (SAMPAIO, 2019).

Os domicílios pesquisados eram compostos por pelo menos um indivíduo na faixa etária de o a 5 anos, e esta variável se mostrou estatisticamente significativa ( $\mathrm{p}=0,02 \mathrm{I})$ para IAN em famílias com crianças menores de três anos de idade. Assim, as prevalências observadas para IAG e IAM, corroboram com os achados no estudo de Ribeiro e colaboradores (2018), onde a presença de morador menor de 18 anos no domicílio, a associação foi observada apenas para Insegurança Alimentar Leve.

Em relação às características de moradia e saneamento, no que se refere a moradia a maior parte das famílias $(76,5 \%)$, informou ter moradia própria, e na questão de cobertura do saneamento básico, $100 \%$ das famílias responderam ter acesso a água tratada, coleta de lixo doméstico e esgotamento sanitário atendidos por meio da rede pública. Este resultado foi superior ao observado em estudo realizado no município de Matuipe e de resultados do CONSAD, onde os dados apontaram para uma deficiente cobertura de saneamento básico, de forma que a exposição familiar a condições adversas de saneamento básico influencia diretamente na saúde da família (RIBEIRO et. al., 20I8; PANELLI-MARTINS, 2008).

No que concerne o saneamento e SAN, este estudo teve como resultado a variável saneamento como fator de proteção para a (in)segurança alimentar. Entretanto, se faz necessário mencionar que os achados neste estudo não condizem com a grande realidade brasileira, onde as desigualdades sociais e acesso inadequado a serviços básicos coexistem (SANTOS et. al., 2018a; WALSH, ROOYEN; 2015).

Verifica-se ainda, no que concerne as variáveis sócio demográficas, o qual avaliou indicadores referentes ao tipo de moradia e renda familiar, que não houve correlação destas variáveis com a prevalência de IAN. Os resultados referentes a renda familiar mensal das famílias estudadas demonstraram que 69,12\% das famílias recebiam um ou mais de um salário mínimo, e 30,88\% tinham renda menor ou igual a um salário mínimo $\left(<\mathrm{R}_{\$} \mathrm{I.045}, 00\right)$.

A IA, aferida pela disponibilidade de recursos financeiros para a compra de alimentos, está associada às dificuldades enfrentadas para uma alimentação saudável. Isso 
porque, em estudo conduzido com usuários de Unidades Básicas de Saúde da zona urbana da cidade de Pelotas, mais da metade da população estudada relatou como principal barreira o elevado custo dos alimentos saudáveis. Além disso, pode-se observar que quanto maior a renda, menor era a probabilidade de ter dificuldades, uma vez que o padrão alimentar de uma população é influenciado pelo seu poder aquisitivo (LINDEMANN; OLIVEIRA; MENDOZA-SASSI, 2016).

Ainda relacionado a renda mensal familiar, neste estudo esta variável não se mostrou relevante para o fenômeno estudado. Este achado difere de outros estudos conduzidos por diversos autores comprovaram que quanto menor a classe de rendimento mensal domiciliar, maior a proporção de domicílios em situação de IAN (RIBEIRO et. al., 20I8; IBGE, 20Ioc; PIMENTEL; SALLES-COSTA, 2009; SOUZA, 2004b).

O efeito do rendimento familiar é substancial sobre a maioria dos alimentos, segundo a POF 2008-2009 (IBGE, 20I0c) que aponta que conforme se reduz a renda, o acesso ao leite e seus derivados, assim como o acesso a frutas, legumes, verduras e carnes diminui.

A percepção sobre as características do ambiente alimentar atua como importante determinante das escolhas alimentares, uma vez que, diferenças de acesso à alimentação saudável entre locais de maior e menor nível socioeconômico podem trazer aos indivíduos diferentes possibilidades para a aquisição destes alimentos. Estudo realizado em São Paulo sobre as relações entre o ambiente e as práticas alimentares em diferentes contextos socioeconômicos e de acesso à alimentação observou que para os indivíduos que viviam em locais caracterizados pelo menor nível de renda, as representações sobre o acesso a estabelecimentos de comercialização de alimentos (feiras, sacolões, supermercados e mercados), mostram carências em relação a disponibilidade, acessibilidade e qualidade destes (ALMEILDA, 2015).

Mister que o simples aumento da disponibilidade de opções mais saudáveis nos ambientes alimentares, não garantirá sua compra e consumo, especialmente entre grupos de baixa renda, se esses alimentos não forem financeiramente acessíveis (SANTOS et al; 2017b).

Neste estudo, famílias beneficiadas e não beneficiadas por programa do governo (Bolsa Família), não atingiram significância estatística para IAN. Corroboram com nossos achados, vários estudos que avaliaram a participação no PBF e a prevalência de IAN em 
famílias com crianças menores de três anos, aferindo uma relação entre o recebimento de benefícios governamentais e a redução da IAN em famílias com crianças (CORREIA et al., 20I8; GODRICH et al., 2017; LUND et al., 2017; SHAMAH-LEVY et al., 2017; SABÓIA; SANTOS, 2015c; FERREIRA et al., 2014; MABLI et al., 2013; VIANNA; SEGALLCORREAA, 2008b).

Entretanto, resultados diferentes foram encontrados em pesquisa do CONSAD Vale do Jiquiriçá, onde foram observados que 68,4\% dos participantes eram contemplados com o benefício do Programa Bolsa Família (PBF); e destes, 45,9\% se encontravam em IAN (RIBEIRO et. al., 2018), corroborando com resultados encontrados em outros estudos onde apontam indícios de relação significante entre IA e o recebimento de benefícios de programas governamentais (SHAMAH-LEVY et al., 2017; GODRICH et al., 2017; LUND et al., 2017).

Assim, tem-se que os programas de transferência de renda foram criados no Brasil como uma estratégia de complementação de renda em face da situação de desemprego e baixa renda de parcelas significativas da população brasileira. Do ponto de vista das políticas públicas, o programa é importante para melhorar as condições de vida das famílias, embora, por si só, não garanta índices satisfatórios de segurança alimentar, questão associada a um quadro de pobreza mais amplo (IBASE, 2008).

Verificou-se ainda, no que concerne a variáveis sócio demográficas, a variável número de moradores revelou que 63,16\% eram constituídas por cinco a seis membros, onde esta variável se mostrou com maior probabilidade de IAN nas famílias ( $p=0,032)$. Nossos resultados vão de encontro com outros estudos que mostram associação entre a IAN e família grande, onde esta é uma das muitas variáveis consideradas na análise de IA, com relação direta com o número de moradores no domicílio, tendo sido uma variável com força explicativa para a insegurança alimentar em vários estudos (SAMPAIO, 20I9; RIBEIRO et. al., 2018; SOUZA, 2009a; ANSCHAU, 2008), em destaque para IAN em categorias mais graves em famílias com número elevado de moradores em especial nas famílias com mais moradores menores de 18 anos (FERREIRA et al., 2014; SU PEI; APPANNAH; SULAIMAN, 2018).

Corroboram com estes dados vários estudos onde relatam que os domicílios com maior densidade domiciliar foram mais propensos à insegurança alimentar moderada e 
grave do que outros domicílios de referência. Isso é esperado, visto que a aglomeração intradomiciliar implica em maior número de moradores para serem alimentados, independente da renda (RIBEIRO et. al., 20I8; OMUEMU; OTASOWIE; ONVIRIUKA, 2012);

Diante desse cenário, é notório que essas transformações nos padrões de consumo tiveram efeitos nos sistemas alimentares especialmente no que tange a sua sustentabilidade. Um sistema alimentar sustentável é aquele que além de fornecer alimentos nutritivos e acessíveis para todos, também possibilita a preservação dos recursos naturais e dos ecossistemas para atender as necessidades humanas atuais e futuras (FAO, OPAS, 2017).

A FAO e a OPAS (2017), em recente relatório reforçam que para pensar nos determinantes de consumo alimentar das famílias há de se considerar quais são os alimentos disponíveis nos mercados alimentares e se a população tem acesso a eles, se são adequados para sua inclusão na dieta, e ainda, se a população está disposta a incluí-los em seu padrão de consumo. Sem a existência de uma oferta variada e acessível de alimentos de diferentes espécies, será impossível para os países promoverem mudanças nos padrões de consumo que permitam o estabelecimento de uma ampla alimentação saudável.

Assim, após diversas ponderações acerca das multideterminações das escolhas alimentares, sejam elas relativas à população adulta ou infantil, é mister que sistemas alimentares mundiais devem proporcionar hábitos alimentares sustentáveis, não apenas do ponto de vista da segurança e da viabilidade econômica, mas também que promovam saúde, equidade e sustentabilidade ambiental (SWINBURN et al, 2015).

\section{CONSIDERAÇÕES FINAIS}

A prevalência de insegurança alimentar e nutricional moderado/grave foi encontrada em uma parcela das famílias pesquisadas, associado este fato ao não acesso a alimentos em quantidade e qualidade adequadas ao consumo, fator este relacionado com o poder aquisitivo das famílias, indicando que a redução quanti-qualitativa a alimentação representa um grande desafio não somente para os formuladores de políticas públicas, mas também para as famílias

Contudo, a interpretação desses resultados não nos permite apontar causas, entretanto apresenta subsídios para pensar a discussão do tema de forma mais efetiva e assim 
servir de orientação a gestão pública no território e afins sobre a direção das ações necessárias, considerando-se as necessidades e limitações diante dessa situação.

\section{REFERENCIAS}

ALMEIDA, Luara Bellinghausen. $\mathrm{O}$ ambiente alimentar, os indivíduos e suas práticas: um estudo no município de São Paulo. o6/II/2015. 254p. Tese de Doutorado em área de Nutrição em Saúde Pública -Departamento, Programa de Pós-Graduação em Nutrição e Saúde Pública, São Paulo, II/2015.

ANSCHAU, Franciele Richetti. Insegurança alimentar de beneficiários de programas de transferência de renda. 30/oI/2008. I07p. Dissertação de Mestrado em área de Saúde Coletiva -Departamento, Programa de Pós-Graduação em Saúde Coletiva, Londrina, or/20o8.

ANTUNES, Natalia Jurgensen. Obesidade infantil: vivências familiares relativas ao processo de aconselhamento nutricional. 21/o8/2018. 223p. Dissertação de Mestrado em área de Politica, Gestão e Saúde -Departamento, Programa de Pós-Graduação em Saúde Pública, São Paulo, o8/2018.

ARAÚJO, Thiago Santos de; OLIVEIRA, Cristieli Sérgio de Menezes; MUNIZ, Pascoal Torres; SILVA-NUNES, Mônica da; CARDOSO, Marly Augusto. Desnutrição infantil em um dos municípios de maior risco nutricional do Brasil: estudo de base populacional na Amazônia Ocidental Brasileira. Revista Brasileira de Epidemiologia. 19(3): 554-566, 2016. BEZERRA, Pollyana Brandão. Relação entre estado nutricional e funcional em crianças com paralisia cerebral. 23/02/2017. 83p. Dissertação de Mestrado em área da Ciências da Saúde Centro, Programa de Pós-Graduação em Saúde da Criança e adolescente, Pernambuco, $02 / 2017$.

BRASIL. Instituto Brasileiro de Geografia e Estatística. Pesquisa de Orçamentos Familiares 2008-2009: antropometria e estado nutricional de crianças, adolescentes e adultos do Brasil. Rio de Janeiro; 2010.

BRASIL. Ministério da Saúde (MS). Curvas de crescimento e classificação do estado nutricional. Brasília, 2008b. [Internet]. [acessado 2012 nov 12]. Disponível em: http://nutricao.saude.gov.br/documentos/ sisvan_norma_tecnica_criancas.pdf BRASIL. Ministério da Saúde. Secretaria de Atenção à Saúde. Departamento de Atenção Básica. Orientações para a coleta e análise de dados antropométricos em serviços de saúde: 
Norma Técnica do Sistema de Vigilância Alimentar e Nutricional - SISVAN. ı ed. Brasília, 20II, 72p. Disponível em: https://bvsms.saude.gov.br/bvs/publicacoes/orientacoes_coleta_analise_dados_antropome tric. Acesso em: or ago. 2015.

BRASIL. Ministério da Saúde. Secretaria de Atenção à Saúde. Orientações para a coleta e análise de dados antropométricos em serviços de saúde: Norma Técnica do Sistema de Vigilância Alimentar e Nutricional - SISVAN. Brasília: Ministério da Saúde;-2oII.

CORREIA, Luciano Lima; HERMANO, Alexandre Lima Rocha; LEITE, Álvaro Jorge Madeiro; SILVA, Anamaria Cavalcante; CAMPOS, Jocileide Sales; MACHADO, Márcia Maria Tavares; LINDSAY, Ana Cristina; CUNHA, Antonio Jo'se Ledo Alves. The relation of cash transfer programs and food insecurity among families with preschool children living in semiarid climates in Brazil. Cadernos Saúde Coletiva. v. 26, n. I, p. 53-62, 2018.

COTTA, Rosangela Minardi Mitre; MACHADO, Juliana Costa. Programa bolsa família e segurança alimentar e nutricional no Brasil: revisão crítica da literatura. Revista Panam Salud Publica. v. 33, n. I, p. 54-60.2013

DAWALIBI, Nathaly Wehbe; ROMANO, Nádia; MARON, Caroline Féres; GOULART, Rita Maria Monteiro; SEKIYA, Denise Ruri Utsunomia. Evaluation of nutritional condition of children assisted in a philanthropic institution, according to the reference standards by $\mathrm{NCHS} / 1977, \mathrm{CDC} / 2000$ and $\mathrm{WHO} / 2006$. Nutrire Revista Sociedade Brasileira Alimentar Nutrição. v. 3, n. 36, p. 49-62, 2011.

FACCHINI, Luiz Augusto; NUNES, Bruno Pereira; MOTTA Janiana Vieira dos Santos; TOMASI Elaine; SILVA Sueli Manjourany; THUMÉ, Elaine; SILVEIRA, Denise Silva; DILÉLIO, Alitéia Santiago; SAES, Mirelle de Oliveira; MIRANDA Vanessa Iribarrem Avena; VOLZ, Pâmela Moraes; OSÓRIO, Alessander; FASSA, Anaclaudia Gastal. Insegurança alimentar no Nordeste e Sul do Brasil: magnitude, fatores associados e padrões de renda per capita para redução das iniquidades. Caderno Saúde Pública. 30(I):I6I-174, 2014. FERREIRA, Haroldo da Silva; CESAR, Juraci Almeida; ASSUNÇÃO, Monica Lopes; HORTA, Bernardo Lessa. Time trends (1992-2005) in undernutrition and obesity among children under fi ve years of age in Alagoas state, Brazil. Caderno Saude Publica. 29:79380o, 2013. 
FERREIRA, Haroldo da Silva; SOUZA, Maria Eduarda Di Cavalcanti; MOURA, Fabiana Andréa; HORTA, Bernardo Lessa. Prevalência e fatores associados à Insegurança Alimentar e Nutricional em famílias dos municípios do norte de Alagoas, Brasil, 2010. Ciência \& Saúde Coletiva. v. 19, n. 5, p. 1533-1542, 2014.

FAO. FOOD AND AGRICULTURE ORGANIZATION. The State of Food Security and Nutrition in the World: Building resilience for Peace and food security. Rome: FAO, 2017. $132 \mathrm{P}$.

GODRICH, Stephanie; LO, Johnny; DAVIES, Christina; DARBY, Jill; DEVINE, Amanda. Prevalence and socio-demographic predictors of food insecurity among regional and remote Western Australian children. Australian and New Zealand Journal of Public Health, v. 4I, n. 6, p. 585-590, 2017.

GUERRA, Lúcia Dias da Silva. Análise da insegurança alimentar e nutricional e fatores associados em domicílios com adolescentes de municípios da área de abrangência da BR I63

- Mato Grosso, Brasil. 20II. 174p. Dissertação de Mestrado em área da Saúde Coletiva Departamento, Programa de Pós-Graduação em Saúde Coletiva, Cuiabá, 201 .

HAWKES, Corinna. Marketing food to children: changes in the global regulatory environment 2004-2006. WHO Library Cataloguing-in-Publication Data, 2007.

IBASE. Balanço social, dez anos: o desafio da transparência / [texto Ciro Torres, Cláudia Mansur]. - Rio de Janeiro: IBASE, 2008. ISBN 978-85-89447-I7-I.

IBGE. Instituto Brasileiro de Geografia e Estatística - IBGE. Pesquisa Nacional por Amostra de Domicílio. Segurança alimentar 2014.

IBGE. Instituto Brasileiro de Geografia e Estatística - IBGE. Pesquisa de orçamentos familiares 2008-2009: antropometria e estado nutricional de crianças, adolescentes e adultos no Brasil. Rio de Janeiro: IBGE; 2010.

LINDEMANN, Ivana Loraine; OLIVEIRA, Riceli Rodeghiero; MENDOZA-SASSI, Raúl Andres. Difficulties in ensuring a healthy diet among primary health care users and associated factors. Ciênc Saúde Coletiva. v. 21, n. 2, p. 599-6ro, 2016. Available from: Disponível em: http://www.scielo.br/pdf/csc/v2In2/I413-8I23-csc-2I-02-0599.pdf. LUND, Thomas B; HOLM, Lottle; TETENS, Inge; SMED, Sinne; NIELSEN, Annemett L. Food insecurity in Denmark-socio-demographic determinants and associations with 
eating- and health-related variables. European Journal of Public Health. v. 28, n. 2, p. 283288, 2017. Disponível em: https://academic.oup.com/eurpub/article/28/2/283/410307o?gclid=CjwKCAjw49qKBhAo EiwAHQVTo2Rp6hiZhoe_dG_VoCwfNnkwFObblOwe_WLSpZN7c6o6laTFLEYdaxoC LGcQAvD_BwE

MABLI, James, et. al,. Measuring the Effect of Supplemental Nutrition Assistance Program (SNAP) Participation on Food Security. United States Department of Agriculture, n. August, 2013, p. 356.

MARTINS, Marlos Melo; PRATA-BARBOSA, Arnaldo; CUNHA, Antonio José Ledo Alves da. Arboviral diseases in pediatrics. J Pediatr (Rio J). v. 96, n. Si, p.2-II, 2020. https://doi.org/ro.1016/j.jped.2019.08.005

MOBLI, Mobli; BANINASAB, Bahram. Effect of indolebutyric acido $n$ root regeneration and seedling survival after transplanting of three Pistacia species. Journal of fruit and ornamental plant research. v.17. n.I. p. 5-13, 2009. Disponível em: http://www.inhort.pl/files/journal_pdf/journal2009/voli7(I)2009/Fullı\%202009_I_pdf.

OLIVEIRA, Juliana Souza; LIRA, Pedro Israel Cabral de; ANDRADE, Sonia Lucia Lucena Souza de; SALES, Aline Cabral; MAIA, Sandra Regina; BATISTA-FILHO, Malaquias. Insegurança alimentar e estado nutricional de crianças de São João do Tigre, no semiárido do Nordeste. Rev Bras Epidemiol. v. 12, n. 3, p. 413-423, 2009. https://doi.org/10.1590/Si415$790 \mathrm{X} 2009000300010$.

OMUEMU, Vivian O; OTASOWIE, Efosa M; ONYIRIUKA, Ugochukwu. Prevalence of food insecurity in Egor local government area of Edo State, Nigeria. Ann Afr Med . v. II, p.139-45, 2012. https://doi.org/10.4103/1596-3519.96862

ONU - Organización de las Naciones Unidas para la Alimentación y la Agricultura. Fondo Internacional de Desarrollo Agrícola. Organización Mundial de la Salud. Fondo de las Naciones Unidas para la Infancia. Programa Mundial de Alimentos. El estado de la seguridad alimentaria y la nutrición en el mundo 2017: Fomentando la resiliencia en aras de la paz y la seguridad alimentaria Roma: FAO; 2017.

ONU. Organização das Nações Unidas. Objetivos de Desenvolvimento Sustentável. 2018. 
OPAS. Organização Pan-Americana da Saúde - Brasília: OPAS; 2017. [Acessado em maio de 2020]. Sistemas alimentares e nutrição: a experiência brasileira para enfrentar todas as formas de má nutrição. Disponível em: http://www.cfn.org.br/wpcontent/uploads/2017/og/oms.pdf. [Google Scholar]

PANELLI-MARTINS, Barbara Eduarda. Análise de métodos de avaliação da segurança alimentar e nutricional: uma contribuição à política municipal de SAN. 21/05/2013. 140p. Dissertação de mestrado em alimentos, Nutrição e Saúde - Programa, Salvador, 05/2013.

PEDRAZA, Dixis Figueroa; GAMA, Jacqueline Santos da Fonseca Almeida. Segurança alimentar e nutricional de famílias com crianças menores de cinco anos do município de Campina Grande, Paraíba. Revista Brasileira de Epidemiologia. v. 18, n. 4, p. 906-917, 2015. https://doi.org/10.1590/1980-5497201500040018.

PEI, Chong Su; APPANNAH, Geeta; SULAIMAN, Norhasmah. Household food insecurity, diet quality, and weight status among indigenous women (Mah Meri) in

Peninsular Malaysia. Nutr Res Pract. v. I2, n. 2, p. I35-I42, 2018. https://doi.org/10.4162/nrp.2018.12.2.135.

PÉREZ-ESCAMILLA, Rafael; SEGALL-CORRÊA, Ana Maria; MARANHA, Lucia Kurdian; SAMPAIO. Maria de Fatima Archanjo; MARÍN-LEÓN, Leticia; PANIGASSI, Giseli. An adapted version of the U.S. Department of Agriculture Food Insecurity module is a valid tool for assessing household food insecurity in Campinas. Brazil. Journal Nutr. v. 134, n. 8, p. 1923-28, 2004.

PIMENTEL, Patricia Gomes; SICHIERI, Roseli; SALLES-COSTA, Rosana. Insegurança alimentar, condições sócio-econômicas e indicadores antropométricos em crianças da Região Metropolitana do Rio de Janeiro, Brasil. Revista Brasileira de Estudos da População. v. 26, n. 2, p. 83-294, 2009. DOI:10.1590/So102-30982009000200008.d POBlACiON, Ana Paula; MARÍN-LEÓN, Leticia; SEGALl-CORRÊA, Ana Maria; SILVEIRA, Jonas Augusto; TADDEI, José Augusto de Aguiar Carrazedo. Insegurança alimentar em domicílios brasileiros com crianças menores de cinco anos. Caderno de Saúde Pública. v. 30, n. 5, p. 1067-1078, 2014. https://doi.org/10.1590/o102-311X00072713. 
RADIMER, Katthy L; OLSON Christine M; GREENE, Jennifer C; CAMPBELL, Cathy C; HABICHT Jena-Pierre. Understanding hunger and developing indicators to assess it in women and children. Journal of Nutrition Education. v.24, n.I, p.36S-45S, 1992.

RIBEIRO, Joelma Silveira; SANTOS, Sandra Maria Chaves; SOARES, Tania Mara Buranelli; ACCIOLY, Juçara Ana Bastos. Segurança Alimentar e Nutricional: avaliação e fatores determinantes em consórcio de municípios, Bahia, Brasil. Demetra Alimentação

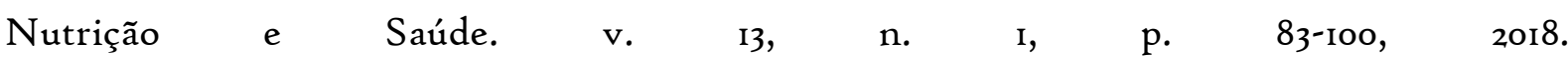
DOI: https://doi.org/10.12957/demetra.2018.300oI.

SABÓIA, R. C. B. DE; SANTOS, M. M. DOS. Prevalência de insegurança alimentar e fatores associados em domicílios cobertos pela Estratégia Saúde da Família em Teresina, Piauí, 2012-2013. Epidemiologia e Serviços de Saúde. v. 24, n. 4, p. 749-758, 2015. https://doi.org/I0.5123/Si679-49742015000400017.

SALLES-COSTA, Rosana; PEREIRA, Rosangela Alves; VASCONCELLOS, Mauricio Teixeira Leite; VEIGA, Gloria Valeria; MARINS, Vânia Maria Ramos; JARDIM, Beatriz Cordeiro; GOMES, Fábio da Silva; SICHIERI, Rosely. Associação entre fatores sócio-econômicos e insegurança alimentar: estudo de base populacional na Região Metropolitana do Rio de Janeiro, Brasil. Revista de Nutrição. v. 2I, n. (Supl.), p.S99-Sıo9, 2008.

Disponível em: https://www.scielo.br/j/rn/a/fq69hBWpxzHC8MFXDfXDLqD/?lang=pt. SAMPAIO, André Cesar Furlaneto. Planejamento Urbano e Meio Ambiente. Maringá-Pr.: UniCesumar, 2019.

SANTOS, Taíse Gomes; SILVEIRA, Jonas Augusto Cardoso; LONGO-SILVA, Giovana; RAMIRES, Elyssia Karine Nunes Mendonça; MENEZES, Risia Cristina Egito. Tendencia e fatores associados á insegurança alimentar no Brasil: Pesquisa Nacional por Amostra de Domicilios 2004, 2009 e 2013. Caderno de Saúde Pública. v. 34, n. 4, p. eooo66917, 2018. https://doi.org/10.159o/oro2-311Xooo66917.

SANTOS, Daniele Ferreira Barbosa; STRAPASSON, Giovanna Chipon; GOLIN, Samantha Daliana Pacheco; GOMES, Eliane Cardoso; WILLE, Grace Maria Ferreira Castro; BARREIRA, Sandra Mara Woranovicz. Implicações da pouca preocupação e percepção familiar no sobrepeso infantil no município de Curitiba, PR, Brasil. Ciência \& 
Saúde Coletiva. v. 22, n. 5, p. 1717-1724, 2017. DOI: https://doi.org/10.1590/141381232017225.13462015.

AZEVEDO, Marta Maria; SEGAlL-COORÊA, Ana Maria; FERREIRA, Maria Beatriz Rocha. Estudo dos conceitos, conhecimentos e percepções sobre segurança, insegurança alimentar e fome em quatro grupos de etnia Guarani no Estado de SP. Campinas: Universidade Estadual de Campinas; p.167-176, 2009. Disponível em: https://www.fef.unicamp.br/fef/sites/uploads/deafa/qvaf/cultura_alimentarcapi7.pdf. SEGALL-CORREAA, A. M. et al. Amamentação e alimentação infantil. In: BRASIL. Ministério da Saúde. Pesquisa Nacional de Demografi a e Saúde da Criança e da Mulher. Brasília, 2009.

SHAMAH-LEVy, Teresa; MUNDO-ROSAS, Verónica; MORALES-RUAN, Carmen; CUEVAS-NASU, Lucia; MÉNDEZ-GÓMEZ-HUMARÁN, Ignacio; PÉREZESCAMILLA, Rafael. Food insecurity and maternal-child nutritional status in Mexico: Cross-sectional analysis of the National Health and Nutrition Survey 2012. BMJ Open. v. 7, n. 7, p. I-II, 2017. DOI: 10.1136/bmjopen-2016-014371.

SOUZA, Luiza de Marilac de. Fatores associados à insegurança alimentar nos domicílios da Região Nordeste do Brasil. II/o2/2009. II3p. Tese de Doutorado em área de Nutrição. Programa de Pós Graduação da Universidade Federal de Minas Gerais; 02/2009.

SANT’ANNA, Mônica de Souza; PRIORE, Silvia Eloíza; FRANCESCHINI, Sylvia do Carmo C. Métodos de avaliação da composição corporal em crianças. Revista Paulista de Pediatria. v.27, n.3, p. 315-321, 2009. DOI: https://doi.org/10.159o/So103-05822009000300013. SPERANDIO, Naiara; MORAIS, Dayane de Castro; PRIORE, Silvia Eloiza. Escalas de percepção da insegurança alimentar validadas: a experiência dos países da América Latina e Caribe. Ciência Saúde Coletiva. v.23, n.2, p. 449-462, 2018. DOI: https://doi.org/I0.1590/141381232018232.08562016 .

SWINBURN, Boyd; KRAAK, Vivica; RUTTER, Harry Rutter; VANDEVIJVERE, Stefanie; LOBSTEIN, Tim; SACKS, Gary; GOMES, Fabio; MARSH, Tim; MAGNUSSON, Roger. Strengthening of accountability systems to create healthy food environments and reduce global obesity. The Lancet. v. 20, n.385(9986), p.2534-45. DOI: 10.1016/Sor40-6736(14)61747-5. 
TRIVELlATO, Paula Torres; MORAIS, Dayane de Castro; LOPES, Silvia Oliveira; MIGUEL, Elisangela da Silva; FRANCESCHINI, Sylvia do Carmo Castro; PRIORE, Silvia Eloiza. Insegurança alimentar e nutricional em famílias do meio rural brasileiro: revisão sistemática. Ciência \& Saúde Coletiva, v.24, n.3, p.865-874, 2019. DOI: https://doi.org/10.1590/1413-81232018243.05352017.

VIANA, Rodrigo Pinheiro Toledo; SEGALL-COSSÊA, Ana Maria. Insegurança alimentar das famílias residentes em municípios do interior do estado da Paraíba, Brasil. Revista de

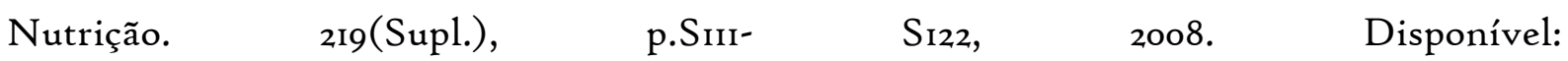
https://www.scielo.br/j/rn/a/CfkM5nMxFm3tZZy83csJm3J/?format=pdf\&lang=pt. WALSH, Corinna; ROOYEN, Francois Cornel. Household food security and hunger in rural and urban communities in the Free State Province, South Africa. Ecology of Food and Nutrition. v.52, n.2, p.I-20, 2014. DOI:10.1080/03670244.2014.964230. 